\title{
FINITE CODIMENSIONAL SUBALGEBRAS OF STEIN ALGEBRAS AND SEMIGLOBALLY STEIN ALGEBRAS
}

\author{
HA HUY KHOAI AND NGUYEN VAN KHUE
}

\begin{abstract}
The following theorem is proved: For each finite codimensional subalgebra $A$ of a Stein algebra $B$ there exists a natural number $n$ such that $B$ is algebraically isomorphic to $A \oplus \mathbf{C}^{n}$.
\end{abstract}

\section{INTRODUCTION}

This paper continues a series of papers on the finiteness of complex analytic spaces. It is well known that for complex Stein spaces a number of problems of complex analysis have solutions: the first Cousin problem, the problem of continuation of holomorphic functions on analytic sets, and so on. In [4] we give a vector space structure to the class of additive Cousin data $\left\{U_{i}, \varphi_{i}\right\}$ on a complex space $X$, and we consider the class of complex spaces for which the set of additive Cousin data having a solution (i.e., the set of additive Cousin data $\left\{U_{i}, \varphi_{i}\right\}, i \in I$, for which there exists a meromorphic function $\varphi$ on $X$ such that $\varphi-\varphi_{i}$ is holomorphic on $U_{i}$ for each $i \in I$ ) is "sufficiently large"; it is a finite codimensional subspace. In [5] we study complex spaces with the following property: for each analytic set $V$ in $X$ the space $\mathscr{O}(X)$ of holomorphic functions on $X$ considered as a subspace of the space $\mathscr{O}(V)$ of holomorphic functions on $V$ is a finite codimensional subspace. In the present paper we consider certain properties of finite codimensional subalgebras of Stein algebras and obtain some corollaries for the continuation of holomorphic functions on Stein subspaces of complex spaces.

First of all we recall some notation and formulate the results of this paper.

A $\mathbf{C}$-algebra is called a Stein algebra if it is algebraically isomorphic to the $\mathbf{C}$ algebra $\mathscr{O}(X)$ of holomorphic functions on a Stein space $X$. For every algebra $B$ we denote by $S(B)$ its spectrum and by $S^{\#}(B)$ the noncontinuous spectrum. It is known that for a Stein algebra $B$ we have

$$
B \cong \mathscr{O}(S(B)) \text { and } S^{\#}(B)=S(B) .
$$

Main Theorem. For each finite codimensional subalgebra $A$ of a Stein algebra $B$ there exists a natural number $n$ such that $B$ is algebraically isomorphic to $A \oplus \mathbf{C}^{n}$.

Received by the editors January 13, 1989 and, in revised form, December 1, 1989.

1980 Mathematics Subject Classification (1985 Revision). Primary 32E25; Secondary 32E10, $30 \mathrm{H} 05$.

The final form of this paper was written while the first author was at the Max-Planck-Institut für Mathematik Bonn. 
There are some corollaries that can be deduced from the Main Theorem but we need some more notation to explain them.

A complete $m$-convex algebra $B$ is said to be a semiglobally weakly holomorphic algebra if it is isomorphic to the algebra $\mathscr{O}(K)$ of holomorphic functions on a weakly holomorphic compact set $K$ (we recall that a compact set $K$ is said to be weakly holomorphic if it has a decreasing neighborhood basis consisting of open sets having an envelope of holomorphy).

Corollary 1. Let $A$ be a finite codimensional subalgebra of a semiglobally weakly holomorphic algebra $B$. Then $B$ is algebraically isomorphic to $\bar{A} \oplus \mathbf{C}^{n}$ for some natural number $n$.

Corollary 2. Let $V$ be a Stein subspace of a complex space $X$, and let $R: \mathscr{O}(X)$ $\rightarrow \mathscr{O}(V)$ be the restriction map. If $\operatorname{dim} \mathscr{O}(V) / R \mathscr{O}(X)<\infty$, then there exists $a$ finite subset $T \subset V$ such that

(i) $V \backslash T$ is an analytic subset of $X$;

(ii) the restriction map $R: \mathscr{O}(X) \rightarrow \mathscr{O}(V \backslash T)$ is surjective.

\section{Proof of the Main Theorem}

2.1. Lemma. Every finite codimensional subalgebra of a Stein algebra is a closed subalgebra.

Remark. It is well known that a finite codimensional subspace of a Banach space must be closed and complemented.

Proof of Lemma 2.1. Let $B$ be a finite codimensional subalgebra of a Stein algebra $B$. Assume that $A \neq \bar{A}$. Take $f \in \bar{A} \backslash A$, and consider the subalgebra $A[f]$ of $\bar{A}$. Since $\operatorname{dim} A[f] / A \leq \operatorname{dim} B / A<\infty$, there exists the minimal polynomial for $f$ :

$$
P(x)=x^{n}+c_{n-1} x^{n-1}+\cdots+c_{1} x+\alpha_{0},
$$

where $c_{1}, \ldots, c_{n-1} \in \mathbf{C}, \alpha_{0} \in A, P(f)=0$. We first prove that the discriminant $D$ of the polynomial $P$ is nonzero. Suppose on the contrary that $D=0$, then for every $\beta \in S^{\#}(B)$ we have

$$
n \hat{f}(\beta)^{n-1}+(n-1) c_{n-1} \hat{f}(\beta)^{n-2}+\cdots+c_{1}=0
$$

where $\hat{f}$ denotes the Gelfand transform of $f$. It follows then that

$$
f^{n-1}+\frac{n-1}{n} c_{n-1} f^{n-2}+\cdots+\frac{c_{1}}{n}=0 \text {. }
$$

This however contradicts the fact that the polynomial $P(x)$ is minimal. This shows $D \neq 0$. Now take $w \in-S^{\#}(A)$ so that $\hat{D}(w) \neq 0$. One finds two complex numbers $\lambda_{1}$ and $\lambda_{2}$ satisfying

$$
\begin{aligned}
& \lambda_{1}^{n}+c_{n-1} \lambda_{1}^{n-1}+\cdots+c_{1} \lambda_{1}+\hat{\alpha}_{0}(w)=0, \\
& \lambda_{2}^{n}+c_{n-1} \lambda_{2}^{n-1}+\cdots+c_{1} \lambda_{2}+\hat{\alpha}_{0}(w)=0 .
\end{aligned}
$$

We define then two multiplicative linear functionals $w_{1}$ and $w_{2}$ on $A[f]$ by the following formulas:

$$
w_{1}\left(\sum_{j=0}^{m} b_{j} f^{j}\right)=\sum_{j=0}^{m} \hat{b}_{j}(w) \lambda_{1}^{j}, \quad w_{2}\left(\sum_{j=0}^{m} b_{j} f^{j}\right)=\sum_{j=0}^{m} \hat{b}_{j}(w) \lambda_{2}^{j} .
$$


It is easy to see that the functionals $w_{1}$ and $w_{2}$ are correctly defined, since if for some polynomial $Q$ we have $Q(f)=0$ then $Q(x)=P(x) R(x)$ and $w_{1}(Q(f))=w_{2}(Q(f))=0$.

Let $I_{1}$ and $I_{2}$ be the integral closures of $\operatorname{ker} w_{1}$ and $\operatorname{ker} w_{2}$ in $B$, respectively. Then we have $I_{1} \cap A[f]=\operatorname{ker} w_{1}$ and $I_{2} \cap A[f]=\operatorname{ker} w_{2} ; I_{1}, I_{2} \neq B$. We let $\widetilde{I}_{1}$ and $\widetilde{I}_{2}$ denote maximal ideals of $B$ which contain $I_{1}$ and $I_{2}$, respectively. Then $B / \widetilde{I}_{1}$ and $B / \tilde{I}_{2}$ are fields which are integral over $A[f] / I_{1}=\mathbf{C}$ and $A[f] / I_{2}=\mathbf{C}$. This implies that $B / \widetilde{I}_{1}=B / \widetilde{I}_{2}=\mathbf{C}$, and hence $\widetilde{I}_{1}=\operatorname{ker} \tilde{w}_{1}$, $\tilde{I}_{2}=\operatorname{ker} \tilde{w}_{2}$, where $\tilde{w}_{1}, \tilde{w}_{2} \in S^{\#}(B)=S(B)$. By the continuity of $\tilde{w}_{1}$ and $\tilde{w}_{2}$ we have $\left.\tilde{w}_{1}\right|_{\bar{A}[f]}=\left.\tilde{w}_{2}\right|_{\bar{A}[f]}$. This contradiction proves Lemma 2.1 .

2.2. Proof in the special case: $S^{\#}(A)$ is normal. Let $R: S^{\#}(B) \rightarrow S^{\#}(A)$ be the restriction map. Since $B$ is integral over $A, R$ is finite, proper and surjective. By Cartan's theorem, $S^{\#}(A)$ has a Stein space structure such that

(i) $A$ is dense in $\mathscr{O}\left(S^{\#}(A)\right)$ (and then, by Lemma 2.1,A= $\bar{A}=\mathscr{O}\left(S^{\#}(A)\right)$ );

(ii) if $f \in \mathscr{O}\left(S^{\#}(B)\right)$ is such that for all $w \in S^{\#}(A)$ one has $\left.f\right|_{R^{-1}(w)}=$ const., then $f \in \mathscr{O}\left(S^{\#}(A)\right)$.

Let $S^{\#}(A)=\amalg Y_{j}$ be the decomposition of $S^{\#}(A)$ into irreducible branches. For every $j$ we denote $X_{j}=R^{-1} Y_{j}$. Then we have

$$
\operatorname{dim} \prod_{j} \mathscr{O}\left(X_{j}\right) / \mathscr{O}\left(Y_{j}\right)=\operatorname{dim} \mathscr{O}\left(S^{\#}(B)\right) / \mathscr{O}\left(S^{\#}(A)\right)<\infty .
$$

This implies that for $j$ sufficiently large we have $\mathscr{O}\left(X_{j}\right)=\mathscr{O}\left(Y_{j}\right)$. Thus, without loss of generality we may assume that $S^{\#}(A)$ is irreducible.

We prove the Main Theorem by induction on $n=\operatorname{codim} A$.

(1) $n=1$. In this case we have $B=\mathbf{C} f+A$ and there exist $\alpha, \beta \in \mathbf{C}$; $g, h \in A$ such that $f^{2}=\alpha f+g, f^{3}=\beta f+h$. This implies that $f^{3}=$ $\beta f+h=\alpha f^{2}+g f=\alpha^{2} f+g f+\alpha g$,

$$
f\left(\alpha^{2}-\beta+g\right)=h-\alpha g .
$$

We consider separately two cases depending on whether or not $\alpha^{2}-\beta+g$ is zero:

(i) $\alpha^{2}-\beta+g \neq 0$. In this case $V\left(\alpha^{2}-\beta+g\right)$ (the analytic set defined by $\left.\alpha^{2}-\beta+g\right)$ is an analytically rare set and we have

$$
\left.f\right|_{S^{*}(B) \backslash R^{-1}\left(V\left(\alpha^{2}-\beta+g\right)\right)}=\frac{h-\left.\alpha g\right|_{S^{*}(A) \backslash V\left(\alpha^{2}-\beta+g\right)}}{\alpha^{2}-\beta+\left.g\right|_{S^{*}(B) \backslash R^{-1}\left(V\left(\alpha^{2}-\beta+g\right)\right)}} .
$$

Thus $f \in \mathscr{O}\left(S^{\#}(A) \backslash V\left(\alpha^{2}-\beta+g\right)\right)$. Since $R$ is proper, $f$ is locally bounded on $S^{\#}(A)$. From the normality of $S^{\#}(A)$ it follows that there exists $\tilde{f} \in \mathscr{O}\left(S^{\#}(A)\right)$ such that $f=\left.\tilde{f}\right|_{S^{*}(A) \backslash V\left(\alpha^{2}-\beta+g\right)}$. On the other hand, since $R^{-1}\left(V\left(\alpha^{2}-\beta+g\right)\right)$ is an analytically rare set, we have $\tilde{f} R=f$. Hence, $f \in \mathscr{O}\left(S^{\#}(A)\right)$, and we have $\mathscr{O}\left(S^{\#}(B)\right)=\mathscr{O}\left(S^{\#}(A)\right)=\bar{A}=A$, which contradicts the hypothesis that $\operatorname{codim} A=1$.

Thus, we have only the following case.

(ii) $\alpha^{2}-\beta+g=0$. In this case $g=\lambda \in \mathbf{C}$, and we have $f^{2}-\alpha f+\lambda=0$. It follows that $f$ takes only two distinct values. We prove that in this case there exists a compact set $K \subset A$ such that the map

$$
R: S^{\#}(B) \backslash R^{-1}(K) \rightarrow S^{\#}(A) \backslash K
$$


is injective. If we assume the contrary, then there exist sequences $\left\{z_{k, 1}\right\}$, $\left\{z_{k, 2}\right\} \subset S^{\#}(B)$ such that $R_{z_{k, 1}}=R_{z_{k, 2}}=t_{k} \rightarrow \infty$ and $z_{k, 1} \neq z_{k, 2}$ for all $k$. Take $u \in \mathscr{O}\left(S^{\#}(B)\right)$ such that $u\left(z_{k, 1}\right)=k, u\left(z_{k, 2}\right)=-k$. Assume that $u=\gamma f+l$, where $\gamma \in \mathbf{C}, l \in A$. Then we have

$$
\gamma\left\{f\left(z_{k, 1}\right)-f\left(z_{k, 2}\right)\right\} \rightarrow \infty .
$$

This contradicts the fact that $f$ takes only two distinct values. Thus there exists a compact set $K$ such that $\left.f\right|_{S^{*}(B) \backslash R^{-1}(K)} \in \mathscr{O}\left(S^{\#}(A) \backslash K\right)$. We denote by $W$ the set of irreducible branches of $S^{\#}(B)$ intersecting with $S^{\#}(B) \backslash R^{-1}(K)$. Then we have $S^{\#}(B)=W \amalg T$, where $T$ is a finite subset of $S^{\#}(B)$. Let $m$ be the number of elements in $T$. We have

$$
B \cong \mathscr{O}\left(S^{\#}(B)\right) \cong \mathscr{O}(W) \oplus \mathbf{C}^{m} \cong \mathscr{O}\left(S^{\#}(A)\right) \oplus \mathbf{C}^{m} \cong \bar{A} \oplus \mathbf{C}^{m}=A \oplus \mathbf{C}^{m}
$$

This completes the proof in the case $n=1$.

(2) $\operatorname{codim} A=n>1$. In this case we have

$$
B=A \oplus \mathbf{C} f_{1} \oplus \cdots \oplus \mathbf{C} f_{n} .
$$

We consider separately two cases: $A\left[f_{1}\right] \neq B$ and $A\left[f_{1}\right]=B$.

(i) $A\left[f_{1}\right] \neq B$. Then we have $\operatorname{dim} A\left[f_{1}\right] / A<\operatorname{dim} B / A=n$.

We consider the restriction map

$$
R_{1}: S^{\#}\left(A\left[f_{1}\right]\right) \rightarrow S^{\#}(A) .
$$

$R_{1}$ is proper and surjective. It follows from the Grauert-Remmert theorem that $S^{\#}\left(A\left[f_{1}\right]\right)$ has Stein space structure such that $A\left[f_{1}\right] \cong \mathscr{O}\left(S^{\#}\left(A\left[f_{1}\right]\right)\right)$. Hence, $A\left[f_{1}\right]$ is a Stein algebra. From the inductive hypothesis it follows that $A\left[f_{1}\right] \cong$ $A \oplus \mathbf{C}^{n_{1}}, B \cong A\left[f_{1}\right] \oplus \mathbf{C}^{n_{2}}$. Thus, $B \cong A \oplus \mathbf{C}^{n_{1}+n_{2}}$.

(ii) $A\left[f_{1}\right]=B$. Since $\operatorname{codim} A<\infty$, there exists an $m$ such that

$$
\begin{aligned}
& f_{1}^{m+1}=\alpha_{m} f_{1}^{m}+\alpha_{m-1} f_{1}^{m-1}+\cdots+\alpha_{1} f_{1}+a_{0} ; \\
& f_{1}^{m+2}=\beta_{m} f_{1}^{m}+\beta_{m-1} f_{1}^{m-1}+\cdots+\beta_{1} f_{1}+b_{0},
\end{aligned}
$$

where $\alpha_{1}, \ldots, \alpha_{m} ; \beta_{1}, \ldots, \beta_{m} \in \mathbf{C} ; a_{0}, b_{0} \in A$. We have

$$
\begin{aligned}
\beta_{m} f_{1}^{m} & +\cdots+\beta_{1} f_{1}+b_{0}=\alpha_{m} f_{1}^{m+1}+\cdots+a_{0} f_{1} \\
= & \alpha_{m}\left(\alpha_{m} f_{1}^{m}+\alpha_{m-1} f_{1}^{m-1}+\cdots+\alpha_{1} f_{1}+a_{0}\right) \\
& +\alpha_{m-1} f_{1}^{m}+\cdots+\alpha_{1} f_{1}^{2}+a_{0} f_{1} .
\end{aligned}
$$

From this it follows that

$$
\begin{gathered}
f_{1}^{m}\left(\beta_{m}-\alpha_{m}^{2}+\alpha_{m-1}\right)+f_{1}^{m-1}\left(\beta_{m-1}-\alpha_{m} \alpha_{m-1}-\alpha_{m-2}\right) \\
+\cdots+f_{1}\left(\beta_{1}-\alpha_{m} \alpha_{1}-a_{0}\right)=-b_{0} .
\end{gathered}
$$

Therefore, a proof similar to that in the case $n=1$ gives us:

$$
B \cong \mathscr{O}\left(S^{\#}(B)\right) \cong A \oplus \mathbf{C}^{k} \text { for some } k .
$$

2.3. Proof in the general case. We denote by $\nu: \widehat{S^{\#}(A)} \rightarrow S^{\#}(A)$ the normalization of $S^{\#}(A)$. Consider the commutative diagram:

$$
\begin{array}{ccc}
Z=S^{\#}(B) \times \widehat{S^{\#}(A)} & \stackrel{\widehat{R}}{\longrightarrow} & \widehat{S^{\#}(A)} \\
S^{\#}(A) & & \\
\downarrow & & \downarrow \nu \\
S^{\#}(B) & \stackrel{R}{\longrightarrow} & S^{\#}(A)
\end{array}
$$


Let $\left\{G_{k}\right\}$ be an increasing exhaustion sequence of holomorphically convex domains in $S^{\#}(A)$. Then $\left\{R^{-1} G_{k}\right\},\left\{\widehat{R}^{-1} \nu^{-1} G_{k}\right\}$, and $\left\{\nu^{-1} G_{k}\right\}$ are exhaustion sequences of holomorphically convex domains in $S^{\#}(B), Z$, and $\widehat{S^{\#}(A)}$, respectively. For each $k \geq 1$ we consider the following exact sequence:

$$
0 \rightarrow \mathscr{O}\left(G_{k}\right) \rightarrow \mathscr{O}\left(R^{-1} G_{k}\right) \rightarrow \mathscr{O}\left(R^{-1}\left(G_{k}\right)\right) / \mathscr{O}\left(G_{k}\right) \rightarrow 0 .
$$

Since for every $k \geq 0$ the restriction map $\mathscr{O}\left(G_{k+1}\right) \rightarrow \mathscr{O}\left(G_{k}\right)$ has a dense image, we have

$$
\mathscr{O}\left(S^{\#}(B)\right) / \mathscr{O}\left(S^{\#}(A)\right)=\lim _{\longleftarrow} \mathscr{O}\left(R^{-1} G_{k}\right) / \mathscr{O}\left(G_{k}\right) .
$$

Therefore there exists a $k_{0}$ such that for all $k \geq k_{0}$ we have

$$
\operatorname{dim} \mathscr{O}\left(R^{-1} G_{k}\right) / \mathscr{O}\left(G_{k}\right)=\operatorname{dim} \mathscr{O}\left(S^{\#}(B)\right) / \mathscr{O}\left(S^{\#}(A)\right) .
$$

From the relation $\widehat{R}^{-1} y=\{x \in S(B) ; R x=\nu y\}=R^{-1} \nu y$ it follows that

$$
\mathscr{O}\left(\hat{\nu}^{-1} R^{-1} G_{k}\right) / \mathscr{O}\left(\nu^{-1} G_{k}\right)
$$

is an integral extension of $\mathscr{O}\left(R^{-1} G_{k}\right) / \mathscr{O}\left(G_{k}\right)$ of degree $\leq \sup \left\{\# R_{y}^{-1}\right\}<\infty$, $y \in S^{\#}(A)$. This implies that there exists $k_{1} \geq k_{0}$ such that for $k \geq k_{1}$ we have

$$
\operatorname{dim} \mathscr{O}\left(\hat{\nu}^{-1} R^{-1} G_{k}\right) / \mathscr{O}\left(\nu^{-1} G_{k}\right)=\text { const. }
$$

By the result of $\S 2.2$ we have

$$
\mathscr{O}\left(\hat{\nu}^{-1} R^{-1} G_{k}\right) \cong \mathscr{O}\left(\nu^{-1} G_{k}\right) \oplus \mathbf{C}^{n}
$$

for some $n$ independent of $k \geq k_{1}$. Therefore we have

$$
\mathscr{O}\left(\widehat{S^{\#}(A)} \oplus \mathbf{C}^{n}\right) \simeq \mathscr{O}(Z) ; \quad Z \cong \widehat{S^{\#}(A)} \amalg\left\{p_{1}, \ldots, p_{n}\right\},
$$

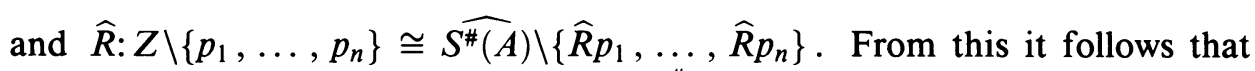
there exists an analytically rare set $V \subset S^{\#}(A)$ such that

$$
R: S^{\#}(B) \backslash R^{-1} V \cong S^{\#}(A) \backslash V .
$$

On the other hand, we have

$$
\operatorname{dim} \mathscr{O}\left(R^{-1} V\right) / \mathscr{O}(V) \leq \operatorname{dim} \mathscr{O}(S(B)) / \mathscr{O}(S(A))<\infty .
$$

By induction we have $\mathscr{O}\left(R^{-1} V\right) \cong \mathscr{O}(V) \oplus \mathbf{C}^{p}$ for some $p>0$. Hence there exist $q_{1}, \ldots, q_{p} \in R^{-1} V$ such that

$$
R: R^{-1} V \backslash\left\{q_{1}, \ldots, q_{p}\right\} \stackrel{\sim}{\rightarrow} V \backslash\left\{R q_{1}, \ldots, R q_{p}\right\} .
$$

From (1) and (2) we obtain

$$
R: S^{\#}(B) \backslash\left\{q_{1}, \ldots, q_{p}\right\} \stackrel{\sim}{\rightarrow} S^{\#}(A) \backslash\left\{R q_{1}, \ldots, R q_{p}\right\} .
$$

Hence we have

$$
B \cong \mathscr{O}\left(S^{\#}(B)\right) \cong \mathscr{O}\left(S^{\#}(A)\right) \oplus \mathbf{C}^{p} \cong \bar{A} \oplus \mathbf{C}^{p}=A \oplus \mathbf{C}^{p} .
$$

The theorem is proved.

\section{PROOF OF THE COROLLARIES}

3.1. Proof of Corollary 1. Let $A$ be a finite codimensional subalgebra of a semiglobally weakly holomorphic algebra $B: B \cong \mathscr{O}(K)$, where $K$ is a weakly 
holomorphic compact set. Take a decreasing neighborhood basis $\mathscr{U}$ of $K$ consisting of open sets having envelope of holomorphy such that $\operatorname{dim}_{x} U>0$ for all $U \in \mathscr{U}$ and $x \in U \backslash K$. Suppose that $B=\mathbf{C} f_{1} \oplus \cdots \oplus \mathbf{C} f_{p} \oplus A$, and set $A_{U}=A \cap \mathscr{O}(U)$. We may assume that $f_{1}, \ldots, f_{p} \in \mathscr{O}\left(U_{1}\right)$. For each $U \in \mathscr{U}$ we denote by $\widehat{U}$ its envelope of holomorphy and by $\delta_{U}, \delta_{\infty}$ the canonical maps: $\delta_{U}: U \rightarrow \widehat{U}, \delta_{\infty}: K \rightarrow S \mathscr{O}(K)$. Then we have $\operatorname{dim} \mathscr{O}(U) / A_{U}=$ $\operatorname{dim} \mathscr{O}(K) / A, \operatorname{dim}_{x} \widehat{U}>0$ for all $U \in \mathscr{U}, U \subset U_{1}$, and $x \in \widehat{U} \backslash \delta_{U} U$. Since $\delta_{U}^{*}: \mathscr{O}(\widehat{U}) \stackrel{\sim}{\rightarrow} \mathscr{O}(U)$ for all $U \in \mathscr{U}$, it is easy to see that $\operatorname{dim}_{x} S \mathscr{O}(K)>0$ for all $x \in S \mathscr{O}(K) \backslash \delta_{\infty} K$. Therefore, by the Main Theorem, there exists a finite subset $\left\{z_{1}, \ldots, z_{p}\right\} \subset K$ such that $\mathscr{O}(U) \cong \bar{A}_{U} \oplus \mathscr{O}\left(\left\{z_{1}, \ldots, z_{p}\right\}\right)$ for all $U \in \mathscr{U}$, $U \subseteq U_{1}$. This implies that

$$
\mathscr{O}(K)=\underset{U \in \mathscr{U}}{\lim _{\vec{U}}} \mathscr{O}(U) \cong \underset{U \in \mathscr{U}}{\lim _{\vec{A}}} \bar{A}_{U} \oplus \mathbf{C}^{p} \cong \bar{A} \oplus \mathbf{C}^{p} .
$$

The corollary is proved.

3.2. Proof of Corollary 2. Let $V$ be a Stein subspace of a complex space $X$, and let $R: \mathscr{O}(X) \rightarrow \mathscr{O}(V)$ be the restriction map and $\operatorname{dim} \mathscr{O}(V) / R \mathscr{O}(X)<\infty$. We set $A=R \mathscr{O}(X)$. By the Main Theorem we have $\mathscr{O}(V) \cong A \oplus \mathbf{C}^{n}$ for some $n$. From this it follows that there exists a finite set $\left\{z_{1}, \ldots, z_{n}\right\} \subset V$ such that $A \cong \mathscr{O}\left(V \backslash\left\{z_{1}, \ldots, z_{n}\right\}\right)$. Corollary 2 is proved.

\section{REFERENCES}

1. H. Grauert and R. Remmert, Theory of Stein spaces, Springer, 1979.

2. R. C. Gunning and H. Rossi, Analytic functions of several complex variables, Prentice-Hall, 1965.

3. Ha Huy Khoai, On the topology of a class of complex manifolds, Proc. 1st Congress Math., Hanoi, 1971.

4. __ Finiteness of complex analytic spaces, Vieinam Math. J. 1 (1973).

5. _ Finite prolongeability of holomorphic functions on analytic sets, Vietnam Math. J. 3 (1973).

Max Planck Institut, D-5300, Bonn, Germany

Institute of Mathematics, P.O. Box 631, Bo Ho 10000 Hanol, Vietnam 